Proyerciones

Vol. 15, N" $V^{\prime \prime} 1$. pp. $91-99$, July 1996

Iniversidad Católica del Norte

Antofagasta - Chile:

\title{
ON COMMUTATIVITY OF RINGS WITH CONSTRAINTS INVOLVING A NIL SUBSET
}

\author{
H. A. S. Abujabal, M. A. Obaid and M. A. Khan \\ King Abdul Aziz University, Saudi Arabia.
}

\begin{abstract}
The main theorem of this paper is that a ring $R$ with unity is ('ommutative if and only if there is a nil subset $B$ of $R$ such that

1. for each $x \in R$, either $x \in Z(R)$ or there is a polynomial $f$ over $\mathrm{Z}$ with $x-x^{2} f(x) \in B$;

2. for each $x, y \in R$, there are non-negative integers $n>1, m, r . s$ depending on a pair of ring elements $x, y$ with $x\left(x^{m} y \pm x^{r} y^{n} x^{4}\right)-$ $\left(x^{m} y \pm x^{r} y^{n} x^{*}\right) x=0$.

$A$ related result for a nil commutative subset of $R$ is given and the restrictions on the hypothesis of our result are justified by examples.
\end{abstract}

AMS Subject classification (1991): 16 U80.

Key words : Commutativity, ring with identity, s-unital rings. 


\section{Introduction}

Throughout, $R$ will denote an associative ring (not necessarily with unity 1), $N(R)$ the set of nilpotent elements of $R . Z(R)$ the center of $R$. and $B$ a non empty subset (not necessarily a subring) of $R$. Z $[\lambda]$ is the totality of polynomials in $\lambda$ with coefficients in $\mathbf{Z}$. the ring of integer's. By $(G F(q))_{2}$, we lnean the ring of $2 \times 2$ matrices over the Galois field $(i F(q)$ with $q$ elements. As usual, $[x, y]$ will denote the commutator $x y-y x$.

Following [8], $R$ is called a left (resp. right) ring s-unit al if for atch $x \in R$ there holds $x \in R x$ (resp. $x \in x R$ ). Furtler, $R$ is called s-unital if it is both left and right s-unital, that is $x \in x R \cap R x$ for each $x \in R$. If $R$ is an s-unital (resp. a left s-unital or a right s-unital) ring, then for any linite subset $F$ of $R$ there exists an element $\epsilon \in R$ such that $\epsilon x=x \epsilon^{\prime}=x$ (resp). $e x=x$ or $x e=x$ ) for all $x \in F$ (see [8, Theorem 1] and [7, Lemma 1]). Such an element $e$ will be called a pseudo-identity (pseudo left-identity or pseudo right-identity) of $F$ in $R$.

In the present paper, we consider the following properties: $(*-B)$ : For each $x$ in $R$, there exists a polynomial $f(\lambda)$ in $\mathbf{Z}[\lambda]$ such that $x-x^{2} f(x) \in B$. (**-B): For each $x$ in $R$, either $x \in Z(R)$, or there exists a polynomial $f(\lambda)$ in $\mathbf{Z}[\lambda]$ such that $x-x^{2} f(x) \in B$. (***-B): For every $a \in B$ and $x \in R,[[a, x], x]=0 .\left(\mathrm{p}_{1}\right)$ : For each $x, y$ in $R$ there exists integers $m=$ $m(x, y) \geq 0, n=n(x, y)>1, r=r(x, y) \geq 0, s=s(x, y) \geq 0$ such that $\left[x, x^{m} y \pm x^{r} y^{n} x^{s}\right]=0 .\left(\mathrm{p}_{2}\right)$ : For each $x, y$ in $R$ there exist integers $m=$ $m(x, y) \geq 0, n=n(x, y)>1, r=r(x, y) \geq 0$ and $s=s(x, y) \geq 0$ such that $\left[x, y x^{m} \pm x^{r} y^{n} x^{s}\right]=0$.

In 195:3, Herstein [4] proved a classical theorem of commutativity of rings satisfying the condition $(*-Z(R))$. Also a nice theorem of Bell [3] establishes commutativity of all rings satisfying $(*-B)$ under some restrictions on $B$. Many authors have studied the commutativity of rings satisfying the property $(*-B)$, but always under some restriction on $B$. Several special cases of $\left(p_{1}\right)$ and $\left(p_{2}\right)$ are known to imply commutativity of rings (see [1], $[2]$ and [9]). For instance, if the integral indices in the underlying comditions are global. The major purpose of this paper is to investigate the commutativity of $R$ when the integral indices are local (i.e. depending on pair of ring elements $x$ and $y$ ). In section 1 of this paper, we explore the commutativity of $R$ when (**-B) holds for a nil subset $B$ together with either of the properties $\left(p_{1}\right)$ and $\left(p_{2}\right)$. Our motivation for studying the commutativity of a ring $R$ satisfying (**-B) for a commutative nil subset $B$ was an attempt to prove a theorem extending a recent result of Tominaga and Yaqub [10] and we present this theorem in section 2. In fact, several 
commutativity theorems can be obtained as a corollaries to our results (see $[1],[2]$ and [5]).

\section{Rings satisfying $\left(p_{1}\right)$ or $\left(p_{2}\right)$ with (**-B) for non-commutative B.}

Theorem 1. If $R$ is a ring with unity 1 , then following statements are equivalent:

(a) $R$ is conmutative

(b) $R$ satisfies the condition $\left(\mathrm{p}_{1}\right)$ and there exists a nil subset $B$ of $R$. for which $R$ satisfies (**-B).

(c) $R$ satisfies the condition $\left(\mathrm{p}_{2}\right)$ and there exists a nil subset $B$ of $R$. for which $R$ satisfies (**-B).

In order to prove our Theorem 1, we state the following well-known lemma:

Lemma 1. Let $R$ be a ring with unity 1 and suppose that $f$ is any polynomial function of two variables on $R$ with the property that $f(x+$ $1, y)=f(x, y)$ for all $x, y$ in $R$. If there exists a positive integer $n$ such that $x^{n} f(x, y)=0$, then $f(x, y)=0$ for all $x, y$ in $R$.

Proof of Theorem 1: Trivially, one can clieck that (a) $\Rightarrow$ (b) and (a) $\Rightarrow$ (c). It is enough to prove that (b) $\Rightarrow$ (a) and (c) $\Rightarrow$ (a). First, we show that $(\mathrm{b}) \Rightarrow(\mathrm{a})$. Let $u \in N(R)$ and $x$ be an arbitrary element of $R$ and let $R$ satisfy $\left(\mathrm{p}_{1}\right)$. Then there exist integers $n_{1}=n(x, u)>1, m_{1}=m(x, u) \geq 0$, $r_{1}=r(x, u) \geq 0$ and $s_{1}=s(x, u) \geq 0$ such that for all $x, y \in R$,

$$
\begin{aligned}
& x^{m_{1}}[x, u]=x^{r_{1}}\left[x, u^{n_{1}}\right] x^{s_{1}}, \\
& x^{m_{1}}[x, u]=x^{r_{1}}\left[u^{n_{1}}, x\right] x^{s_{1}} .
\end{aligned}
$$

Now, let $n_{2}=n\left(x, u^{n_{1}}\right)>1, m_{2}=m\left(x, u^{n_{1}}\right) \geq, r_{2}=r\left(x, u^{n_{1}}\right) \geq 0$ and $s_{2}=s\left(x, u^{n_{1}}\right) \geq 0$. Then

$$
\begin{aligned}
x^{m_{1}+m_{2}}[x, u] & =x^{m_{2}}\left(x^{r_{1}}\left[x, u^{n_{1}}\right] x^{s_{1}}\right) \\
x^{m_{1}+m_{2}}[x, u] & =x^{r_{1}+r_{2}}\left[x, u^{n_{1} n_{2}}\right] x^{s_{1}+s_{2}}
\end{aligned}
$$

and

$$
x^{m_{1}+m_{2}}[x, u]=x^{r_{1}+r_{2}}\left[u^{n_{1} n_{2}}, x \mid x^{s_{1}+s_{2}} .\right.
$$

Hence, for any positive integer $t$, we have integers

$$
n_{1}, n_{2}, \ldots, n_{t}>1, m_{1}, m_{2}, \ldots, m_{t} \geq 0, r_{1}, r_{2}, \ldots, r_{t} \geq 0
$$


and $s_{1}, s_{2}, \ldots, s_{t} \geq 0$ swith that

$$
x^{m_{1} \mid m_{2} ! \ldots+m_{t}}[\ldots, n]=r^{r_{1}+r_{2}+\ldots+r_{1}}\left[\ldots, u^{n_{1} n_{2} \ldots n_{4}}\right] x^{n_{1}+n_{2}+\ldots+m_{4}}
$$

and

$$
\left.x^{m_{1}+m_{2} \mid} \quad\left|m z_{t}[r, u]=x^{T_{1}+r_{2}+\ldots+r_{1}}\right| u^{n_{1} n_{2} \ldots n_{t}}, x\right] x^{s_{1}+s_{2}+\ldots \mid s_{t}} .
$$

But $u^{n_{1} n_{2} \ldots n_{t}}=0$ o for sufficiently large $t$. Therefore, $x^{m_{1}+m, 2+\ldots ; n_{t}}\left[x_{2} u\right]=0$ and by Lemmia $l,[, u, u]=0$ and hence $u \in Z(R)$. Therefore $N(R) \subseteq Z(R)$ and in view of $(* *-13) R$ satisties $(*-\not /(R))$. Thus $R$ is commutative by Herstein's Theorem [4]. Next, let (c) $\Rightarrow$ (a) and $R$ satisfies $\left(p_{2}\right)$. Then by using tane steps as above, we obtain the commutativity of $R$.

The following example shows that it is essential to keep any one of the conditions $\left(\mathrm{p}_{1}\right)$ and $\left(\mathrm{p}_{2}\right)$ together with $(* *-\mathrm{B})$ in the hypotheses of Theorem l to got the commutativity of $n$.

Example 1。Consider

$$
R=\left\{w I+X \mid X=\left(\begin{array}{lll}
0 & 0 & 0 \\
x & j & 0 \\
y & z & 0
\end{array}\right), I==\left(\begin{array}{c}
100 \\
010 \\
001
\end{array}\right)\right.
$$

where $u, x, y, z \in G F(2)\}$.

Trivially, one can ses that $N(R)=\{X\}$ and $R$ satisfies neither ( $\left.p_{1}\right)$ nor $\left(p_{r}\right)$. Let $B=N(R)$. Ther for aliy $x \in R$ such that $\lambda-\lambda^{2} f(\lambda) \in B$. But, R. is non-rommutative.

Remark 1. One might dyacre that in the statenent of condition (p1) and $\left(x_{2}\right)$, the recuirement $n(x, y)>1$ is necessary $i$ cader to prove Theorem 1 since if $R$ is a ring of chanacteristic 2 , then $F$ satisfies $\left(p_{1}\right)$ and $\left(p_{2}\right)$ for global values of $m=r=s=0$ and $n=1$, and Example 1 gives a nom-emmutative ring that satisfies (**-E.).

Fxample 2. Colsider $R=\left\{\left(\begin{array}{ccc}0 & 0 & 0 \\ \alpha & 0 & 0 \\ \beta & \gamma & 0\end{array}\right) \mid \alpha, \beta, \gamma \in G F(2)\right\}$. It is casy to ses that $R$ is in nilpotent ring of incis 3 . Hence $R=N(R)$ and $R$ satisfics both conditions $\left(p_{1}\right)$ and $\left(\mathrm{p}_{2}\right)$. Further, let $B=N(R)$. Then $R$ atso sat isties $(* *-\mathrm{B})$. However, $R$ is non-commutative.

Remark 2. Fixanple ? shows that the existence of unity is essential in the ypothesis of Theor m 1. 
Remark 3. The following example rules out the possibility that $n(x, y)=1$ in Theorem 1.

Example 3. Let $G=\left\langle\epsilon_{1}, \epsilon_{2}, \ldots ; \epsilon_{i}^{2}=0, \epsilon_{i} \epsilon_{j}+\epsilon_{j} \epsilon_{i}=0\right\rangle$ be a Grassman algebra. Set $x=\epsilon_{1}$ and $y=\epsilon_{2}$ with $\epsilon_{1}^{2}=0$ and $\epsilon_{1} \epsilon_{2}+\epsilon_{2} \epsilon_{1}=0$. It can easily be verified that $\left(i\right.$ satisfies $\left(p_{1}\right)$ and $\left(p_{2}\right)$ for all non-negative values of $m, r, s$ and $n=1$. But, $G$ is non-commutative with unity.

Now, Theorem 1 may be generalized as follows:

Theorem 2. Let $R$ be a left s-unital ring satisfying ( $\mathrm{p}_{1}$ ) and suppose there exists a subset $B$ of $N(R)$ for which $R$ satisfies (**-B). Then $R$ is commutative (and conversely).

Theorem 3. Let $R$ be a right s-unital ring satisfying $\left(\mathrm{p}_{2}\right)$. Suppose, further that there exists a subset $B$ of $N(R)$ for which $R$ satisfies (**-B). Then $R$ is commutative (and conversely). Following Lemmas are essential in proving our theorem.

Lemma 2 [10]. Let $R$ be a left (resp right) s-unital ring. If for each pair of elements $x$ and $y$ in $R$, there exists a positive integer $k=k(x, y)$ and an element $e=e(x, y)$ of $R$ such that $x^{k} e=x^{k}$ and $y^{k} e=y^{k}\left(\operatorname{resp} e x^{k}=x^{k}\right.$ and $e y^{k}=y^{k}$ ), then $R$ is an s-unital ring.

Lemma 3. Let $R$ be a left s-unital ring satisfying $\left(p_{1}\right)$. Then $R$ is an s-unital ring.

Proof. Let $x, y \in R$. Then there exists $e=e(x, y) \in R$ such that $\epsilon x=x$ and $e y=y$. Further, there exist nonnegative integers $n=n(x, e)>1, m=$ $(x, \epsilon), r=(x, \epsilon)$, and $s=s(x, e)>0$ such that $x^{m}[x, \epsilon]= \pm x^{r}\left[x, \epsilon^{n}\right] x^{s}$. So $x^{m+1} \epsilon^{\prime}-x^{m} \epsilon x= \pm\left(x^{r+1} e^{n} x^{s}-x^{r} e^{n} x^{s+1}\right)$. Hence $x^{m+1} \epsilon=x^{m+1}$. Also, if $n_{1}=n(y, \epsilon)>1, m_{1}=m(y, \epsilon), r_{1}=r(y, \epsilon)$ and $s_{1}=s(y, \epsilon)>$ 0 are non-negative integers, then $y^{m_{1}+1} e=y^{m_{1}+1}$. Hence $x^{m+m_{1}+2} \epsilon=$ $x^{m+1}\left(x^{m_{1}+1} c\right)=x^{m+m_{1}+2}$ and $y^{m+m_{1}+2} e=y^{m+m_{1}+2}$. Therefore, $R$ is s-unital by Lemma 2 . Let $s=s(x, y)=0$. Then $x^{m+1} y-x^{m} y x=$ $\pm\left(x^{r+1} y^{n}-x^{r} y^{n} x\right)$. So $e^{m+1} y-\epsilon^{m} y e= \pm\left(e^{r+1} y^{n}-\epsilon^{r} y^{n} e\right)$ and thus $y=y e \pm\left(y^{n}-y^{n} e\right)=y\left(e \pm\left(y^{n-1}-y^{n-1} e\right)\right) \in y R$. Thus $R$ is right sunital and hence $R$ is s-unital.

Lemma 4. Let $R$ be right s-unital ring satisfying $\left(\mathrm{p}_{2}\right)$. Then $R$ is an s-unital ring.

Proofo Let $x, y \in R$. Then there exists $e=e(x, y) \in R$ such that $x \epsilon^{2}=x$ and $y \epsilon=y$. Also, there exists non-negative integers $n=n(x, \epsilon)>1, m=$ $(x, e), r=r(x, e)>0$, and $s=s(x, e)$ such that $x^{r+1}=e x^{r+1}$. Also if 
$n_{1}=n(y, e)>1, r_{1}=r(y, e)>0, m_{1}=m(y, e)$ and $s_{1}=s(y, e)$ are nonnegative integers, then we obtain $y^{m_{1}+1}=e y^{m_{1}+1}$. Hence $e x^{m+m_{1}+2}=$ $x^{m+m_{1}+2}$ and $\epsilon y^{m+m_{1}+2}=y^{m+m_{1}+2}$. Therefore, $R$ is s-unital by Lemma 2 . If $r=r(x, y)=0$, then $(x y-y x) x^{m}= \pm\left(x y^{n}-y^{n} x\right) x^{s}$ and we can easily see that $y=\left(e \mp\left(e y^{n-1}-y^{n-1}\right)\right) y \in R y$ for $n=n(e, y)>1$. Thus $R$ is left s-unital. Hence $R$ is s-unital.

Proof of Theorem 2. Let $R$ be left s-unital ring satisfying $\left(\mathrm{p}_{1}\right)$. Then by Lemma $3, R$ is s-unital. In view of [5, Proposition 1] we may assume that $R$ has unity 1 and thus $R$ is commutative by Theorem 1 .

Corollary 1 [2]。 Let $n>1, m, r$ and $s$ be fixed non-negative integers. If $R$ is a left s-unital ring satisfies $\left[x^{m} y \pm x^{r} y^{m} x^{s}, x\right]=0$ for all $x, y$ in $R$, then $R$ is commutative.

Proof of Theorem 3. Let $R$ be right s-unital ring satisfying $\left(\mathrm{p}_{2}\right)$. Then by Lemma $4, R$ is s-unital. In view of proposition of [5], we may assume that $R$ has unity 1 and so $R$ is commutative by Theorem 1 .

Corollary 2. Let $m>1, n, r$ and $s$ be fixed non-negative integers. If $R$ is a right s-unital ring which satisfies $\left[y x^{m} \pm x^{r} y^{n} x^{s}, x\right],=0$ for all $x, y \in R$, then $R$ is commutative.

Remark 4。Let $m=r=0(\operatorname{resp} m=s=0)$ in $\left(\mathrm{p}_{1}\right)\left(\operatorname{resp} .\left(\mathrm{p}_{2}\right)\right)$. Then

$$
[x, y]= \pm\left[y^{n}, x\right] x^{s} \text { for all } x, y \in R
$$

(resp.

$$
\left.[x, y]= \pm x^{r}\left[y^{n}, x\right] \text { for all } x, y \in R\right) .
$$

Let $n>1$ or $s \geq 1$ in (1) ( $\operatorname{resp} n>1$ or $r \geq 1$ in (2). Then $R$ is a $(\mathbf{Z}, \beta)$-ring in the sense of Streb [11], hence $R$ is commutative even if $R$ is not assumed to be a left (resp. right) s-unital ring (ring with unity 1), (cf [11, Lemma 9]).

Remark 5. The following examples demonstrate that there are noncommutative left (resp. right) s-unital rings satisfying $\left(\mathrm{p}_{1}\right)$ (resp. $\left.\left(\mathrm{p}_{2}\right)\right)$.

Example 4. Consider $S_{1}=\left\{\left(\begin{array}{ll}0 & 0 \\ 0 & 0\end{array}\right),\left(\begin{array}{ll}1 & 0 \\ 1 & 0\end{array}\right),\left(\begin{array}{ll}0 & 1 \\ 0 & 1\end{array}\right),\left(\begin{array}{ll}1 & 1 \\ 1 & 1\end{array}\right)\right\}$ and

$$
S_{2}=\left\{\left(\begin{array}{ll}
0 & 0 \\
0 & 0
\end{array}\right),\left(\begin{array}{ll}
1 & 1 \\
0 & 0
\end{array}\right),\left(\begin{array}{ll}
0 & 0 \\
1 & 1
\end{array}\right),\left(\begin{array}{ll}
1 & 1 \\
1 & 1
\end{array}\right)\right\}
$$

be two subrings of $2 \times 2$ matrices over $G F(2)$. One can easily see that in both cases $N(R)$ is the set consisting of the matrices $\left(\begin{array}{ll}0 & 0 \\ 0 & 0\end{array}\right),\left(\begin{array}{ll}1 & 1 \\ 1 & 1\end{array}\right)$. 
Next, let $B=N(R)$. Then for any $x$ in $R$ we can find a polynomial $f(\lambda)$. for instance, $f(\lambda)=\lambda$ such that $x-x^{2} f(x)$ in $B$. But, $s_{1}$ is a left s-mnital ring that satisfies $\left(\mathrm{p}_{1}\right)$ and $S_{2}$ is a right s-unital ring that satisfies (p) for any $n>1, m, r, s \geq 0$.

\section{Rings satisfying $(* *-\mathbf{B})$ for commutative $B$.}

Onr final theorem, a companion theorem to Theorem 3 of $[10]$ is

Theorem 4. Let $R$ be a normal ring, and let $B$ be a nil commulative subset of $R$ for which $R$ satisfies (**-B). Then $R$ is commutative. Before beginning the general proof, we need the following lemma which proved in $[10]$.

Leimma 5. (i) Let $\phi$ be a ring homomorphism of $R$ onto $R^{*}$. If $R$ satisfies $(*-\mathrm{B}),(* *-\mathrm{B})$ or $(* * *-\mathrm{B})$, then $R^{*}$ satisfies $(*-\phi(B)),(* *-\phi(B))$ or $(* * *-\phi(B))$ respectively. (ii) If $B$ is commutative and $R$ satisfies (**-B), then $N(R)$ is a commutative nil ideal of $R$ and contained in a centralizer of $B$, in particular $N(R)^{2} \subseteq Z(R)$. (iii) If there exists a commutative subset $B$ of $N(R)$ for which $R$ satisfies (**-B) and (***-B), then $R$ is commutative.

Proof of Theorem 4. By Lemma 5(i) $R$ assumed to be subdirectly irreducible. If $x$ is an arbitrary element in $R / Z(R)$, using (**-B), we obtain that there exists $y \in\langle x\rangle$ and a positive integer $n$ such that $x^{n}=x^{n+1} y$. Trivially, $e=x^{n} y^{n}$ is an idempotent with $x^{n}=x^{n} e$. But idempotents of $R$. are central, thus $\epsilon$ is either 0 or 1 . Since $R$ has no unity then $\epsilon=0$ and by lemma 5 (ii) $x$ is in the commutative ideal $N$ and so $[[a, x], x]=0$ for all $a \in B$. Thus $R$ is commutative by Lemma 5 (iii).

Remark 6. The above example 4 shows that the property (**-B) alone does not imply commutativity of rings in Theorem 4.

In this regards one might gives the following:

Conjecture. If $R$ is a ring satisfies any one of the properties $\left(p_{1}\right)$ and $\left(p_{2}\right)$. Moreover, if there exists a nil commutative subset $\mathrm{B}$ for which $R$ satisfies (**-B), then $R$ is commutative.

Remark 7. From the proof of Theorem 4, we can observe that the above conjecture is true when $R$ is normal. Example 4 shows that the above (onjecture is not true because the centrality of idempotent in $S_{1}$ (resp $S_{2}$ ) are not followed by $\left(\mathrm{p}_{1}\right)$ (resp. $\left(\mathrm{p}_{2}\right)$ ) together with $(* *-\mathrm{B})$.

Acknowledgement. The authors would like to express there sincere thanks to the referee for the valuable suggestions which help to clarify and simplify the whole paper. In particular, Remark 1 is due to the referee. 


\section{References}

[1] H. A. S. Abujabal and M. A. Khan, " Commutativity of one sided s-unital rings," Internat. J. Math. and Math. Sci., 15 , pp. 815$818,199^{\circ}$.

[2] H. A. S. Abujabal, M. A. Khan and M. S. Samman, "On commutativity of one-sided s-unital rings," Tankang J. Math., 2:3 , pp. $253-258,1992$.

[3] H. E. Bell, "On two commutativity properties for rings," Mat. Japonica, 26 , pp. $523-528,1981$.

[4] I. N. Herstein, "The structure of certain class of rings," Aner. J. Math., 75 , pp. $864-871,1953$.

[5] Y. Hirano, Y. Kobayashi and H. Tominaga, " Some polynomial identities and commutativity of s-unital rings," Matl. J. Okayama Iniversity, 24 , pp. $7-13,1982$.

[6] Y. Hirano, M. Hongan and H. Tominaga, "Commutativity theorems for certain rings," Math. J. Okayama University, 22 , pp. $65-$ $72,1980$.

[7] I. Mogami and M. Hongan, "Note on conmutativity of rings," Matl. J. Okayama University, 20 , pp. $21-24,1978$.

[8] H. Tominaga, "On s-unital rings," Math. J. Okayama University, 18 , pp. $117-134,1976$.

[9] H. Tominaga and A. Yaqub, "Some commutativity properties for rings II," Math. J. Okayama University, 25 , pp. 173 - 179, 198:3.

[10] H. Tominaga and A. Yaqub, "A commutativity theorem for one sided s-unital rings," Math. J. Okayama University, 25, pp. $125-$ $128,1984$.

[11] W.Streb, "Zur Struktur Nichtommutativer ringe", Math. J. Okayama University, 31 ), pp. $135-140,1989$. 
Received : December 05, 1995.

H。 A。 S. Abujabal

M. A. Obaid

and

M. A. Khau

Department of Mathematics

Faculty of Science

King Abdul University

P. O. BOX :31.161

Jectctals 21497

SALDI ARABIA 